\title{
Die Wissensgrundlagen des eigenen Fachs verstehen - empirische Befunde zu den epistemologischen Orientierungen von Lehrkräften geisteswissenschaftlicher Fächer
}

\author{
Caroline Rau ii
}

Eingegangen: 4. März 2020 / Überarbeitet: 14. Dezember 2020 / Angenommen: 17. Januar 2021 / Online publiziert: 5. Februar 2021

(C) Der/die Autor(en) 2021

Zusammenfassung Welche handlungsleitenden Orientierungen zeigen Lehrkräfte, die ein geisteswissenschaftliches Fach unterrichten, hinsichtlich der epistemologischen Grundlagen ihres Faches? Diese Frage steht im Mittelpunkt der hier vorgestellten Studie. Die Forschungsarbeit ist im qualitativ-rekonstruktiven Paradigma verortet: Die Daten wurden mittels Gruppendiskussionsverfahren erhoben und mit der Dokumentarischen Methode ausgewertet. Es konnten drei Idealtypen rekonstruiert werden: In ihnen wird sichtbar, dass Lehrkräfte Unterrichtsinhalten Gültigkeit anhand unterschiedlicher Modi verschaffen. Die Befunde werden vor dem Hintergrund erkenntnistheoretisch-hermeneutischer sowie kulturtheoretischer Gesichtspunkte diskutiert.

Schlüsselwörter Epistemologie - Lehrerprofessionalität · Kulturvermittlung · Geisteswissenschaften · Hermeneutik

Dr. C. Rau $(\bowtie)$

Lehrstuhl für Allgemeine Pädagogik, Otto-Friedrich-Universität Bamberg,

Markusplatz 3, 96047 Bamberg, Deutschland

E-Mail: caroline.rau@uni-bamberg.de 


\title{
Understanding the knowledge base of their own subject-empirical findings on the epistemological orientations of teachers of humanities subjects
}

\begin{abstract}
The study reconstructs the orientations that guide humanities teachers with regard to the epistemological foundations of their subject. This question is the focus of the study presented here. The study is based on the qualitative-reconstructive research paradigm: The data were collected in group discussions and analysed using the documentary method. They choose different modes to produce validity; the study identifies three ideal types of engagement. The findings are discussed against the background of epistemological-hermeneutical and cultural-theoretical aspects.
\end{abstract}

Keywords Epistemology · Teacher professionalism · Teaching culture · Humanities · Hermeneutics

\section{Einleitung}

Lehrkräfte vermitteln im schulischen Kontext vielfältige Vorstellungen über geisteswissenschaftliche Wissensbestände. Mittlerweile liegt eine Fülle von Untersuchungen dazu vor, wie Lehrkräfte der mathematisch-naturwissenschaftlichen Fächer deren epistemologische Grundlagen konzeptualisieren. Diese Frage wurde jedoch bisher für Lehrkräfte der geisteswissenschaftlichen Fächerdomäne kaum in den Blick genommen. An dieses Forschungsdesiderat knüpft der vorliegende Beitrag an. Untersucht werden die handlungsleitenden Orientierungen von Lehrkräften, die ein geisteswissenschaftliches Fach unterrichten. Im Mittelpunkt steht dabei die Frage, wie in dieser Domäne die epistemologischen Grundlagen von Lehrkräften konzeptualisiert werden.

Dazu werden im Folgenden zunächst die erkenntnistheoretischen Anforderungen skizziert, die mit der Genese und Konstruktion geisteswissenschaftlichen bzw. kulturbezogenen Wissens verbunden sind (vgl. Abschn. 2). In Abschn. 3 wird der Forschungsstand $\mathrm{zu}$ denjenigen Facetten der Lehrprofessionalität konturiert, die sich auf epistemologische Fragen beziehen. Im Mittelpunkt dieses Beitrags steht die empirische Untersuchung von Lehrkräften hinsichtlich ihres Verständnisses von Geisteswissenschaften. In Abschn. 4 werden das methodische Vorgehen und dessen methodologische Anbindung erläutert. In Abschn. 5 werden die empirischen Ergebnisse dargestellt und deutlich gemacht, (a) wie Lehrkräfte geisteswissenschaftlichkulturbezogene Inhalte bzw. Erkenntnisobjekte konstruieren, (b) welche Bedeutung dem Produktions- und Rezeptionskontext kultureller Objektivationen eingeräumt wird und (c) wie Geltung bzw. Objektivität von geisteswissenschaftlichem Wissen gegenüber Schülerinnen und Schüler ausgewiesen wird. Die Befunde werden in Abschn. 6 diskutiert. 


\section{Epistemologische Anforderungen: Zur Genese und Konstruktion geisteswissenschaftlichen Wissens}

Aus fachwissenschaftlicher Hinsicht wird einerseits eine ,verloren gegangene Einheit“ (Reinalter und Brenner 2011, S. VII) der geisteswissenschaftlichen Fächer beklagt. Andererseits wird aus einer ideengeschichtlichen Perspektive der Versuch unternommen, das genuin Gemeinsame der Geisteswissenschaften darzustellen. Als dieses Gemeinsame kommen drei Perspektiven in den Blick:

(1) Die Bestimmung geisteswissenschaftlicher Erkenntnisobjekte Gemeinsam ist es den geisteswissenschaftlichen Fächern, dass es um kulturelle Objektivationen (z. B. Texte, menschliche Äußerungen, mündliche Ausdrucksformen usw.) geht, die ,als Sinnsysteme [...] [und] als sinngebendes Symbolsystem einer Gesellschaft" (Reinalter und Brenner 2011, S. VII) ausgewiesen werden. Kulturelle Objektivationen „als potenzielle Sinnträger“ (Böhme 1994, S. 261) verfügen demnach über „eine Doppelheit von bloßer Gegebenheit und des Woraufhin einer Verweisung“, sodass Sinn auch je nach Verwendungszusammenhang der kulturellen Objektivation bestimmt werden kann. Das erkenntnistheoretische Ziel ist es, dieses Sinngewebe zu (de-)konstruieren. Objektivationen können dabei als eindeutig und bzw. oder als mehrdeutig in ihrem Sinngehalt bestimmt werden: Einerseits wird die Position eingenommen, dass kulturelle Objektivationen über einen z. B. vom Autor gesetzten Sinn verfügen. Andererseits wird die Position vertreten, dass Rezipierende kulturellen Objektivationen einen Sinn bzw. ein Sinngewebe verleihen können (vgl. Brenner 2011a; Veraart und Wimmer 1995). Im Kontext hermeneutischer Diskurse wird die Sinnerfassung in methodologischer Hinsicht in den Blick genommen: Vor diesem Hintergrund wird die Hermeneutik auch als ,Grundlegungstheorie der Geisteswissenschaften“ (Brenner 2011b, S. 317) ausgewiesen.

(2) Zur Bedeutung des Produktions- und Rezeptionskontextes für die Sinnzuschreibung Im Kontext der hermeneutischen Theoriegeschichte wurde die Differenz zwischen Produktions- und Rezeptionskontext kultureller Objektivationen herausgestellt und deren Bedeutung für die Sinnzuschreibung beschrieben. Im Kontext älterer Hermeneutikansätze wurden Rezipierende angehalten, den eindeutigen Sinn, den Autoren einer kulturellen Objektivation verliehen haben, zu reproduzieren. Folgten Lehrkräfte diesem Paradigma, würden sie beispielsweise Schülerinnen und Schüler die Autorenintention von Gedichten herausarbeiten lassen. Im Kontext jüngerer Hermeneutikdiskurse werden die Wirkungsgeschichte, die Applikation, die Perspektivität sowie die subjektiven Wissensbestände von Rezipierenden für die Sinnerfassung und -setzung betont. Damit hat der Rezeptionskontext einen deutlich höheren Stellenwert erfahren. Folgten Lehrkräfte diesem Paradigma, würden sie Schülerinnen und Schüler beispielsweise u. a. folgende Aufgabenstellung bearbeiten lassen: Welche Bedeutung hat dieser Text ganz persönlich für dich?

(3) Zur Objektivität von Geltung geisteswissenschaftlicher Sinnzuschreibungen Im Rahmen der Erkenntnisgenese kann kulturellen Objektivationen ein Sinn bzw. ein Sinngewebe sowohl aus ihrem Produktionskontext heraus als auch 
auf der Basis des Rezeptionskontextes seitens der Rezipierenden zugeschrieben werden. Objektivationen wird eine Pluralität von Deutungsmöglichkeiten attestiert. Während die (subjektive) Perspektivität lange Zeit störend für die Gewährleistung von Objektivität herausgehoben wurde, avancierte sie zunehmend zur obligatorischen Bedingung geisteswissenschaftlicher Erkenntnisgenese. Cornelißen fasst den Diskurs zur Objektivität geisteswissenschaftlicher Wissensbestände zusammen und hebt die intersubjektive Überprüfbarkeit, die Begründung und Rechtfertigung von Erkenntnis hervor. Er pointiert, dass ,,[i]n einem allg. Begriffsverständnis [...] die intersubjektive Überprüfbarkeit von Aussagen sowie von Erkenntnisweisen und Darstellungsformen als Ausweis von Objektivität in den Geisteswissenschaften“ (Cornelißen 2011, S. 595) gilt. Vor dem Hintergrund dieses Anspruchs ginge es dann im Unterricht darum, Verstehenskontexte im Kontext der Erkenntnisgenese zu reflektieren und ggf. zu problematisieren. Schülerinnen und Schüler sowie Lehrkräfte sollten demnach kulturellen Objektivationen insofern reflexiv begegnen, als dass die eigene Subjektivität mögliche Erkenntnis sowohl limitiert als auch erweitert. Die intersubjektive Überprüfung sowie die Begründung und Darstellung der eigenen Lesarten bzw. Sinnzuschreibungen können die Objektivität und die Geltung der Sinnzuschreibung(en) ausweisen.

Mit diesen erkenntnistheoretischen Gemeinsamkeiten geisteswissenschaftlicher Schulfächer wie Geschichte, Philosophie, Ethik, Religion oder Deutsch sind Anforderungen skizziert, denen sich Lehrkräfte fachlich zu stellen haben.

\section{Forschungsstand}

Diese fachbezogenen erkenntnistheoretischen Facetten der Lehrerprofessionalität werden im Allgemeinen unter dem Konzept der ,epistemologischen Überzeugungen“ diskutiert. Da epistemologische Überzeugungen einen großen Einfluss darauf nehmen, wie der Welt begegnet bzw. wie diese (vor-)strukturiert wird, entfalten sie Wirkungsmächtigkeit. Ihr Einfluss wird evidenzbasiert hoch eingeschätzt, da sie kognitive Denkleistungen, Motivation sowie Informationsverarbeitungsprozesse beeinflussen (vgl. Bråten 2016; Bromme et al. 2016; Buehl und Fives 2016; Hofer und Bendixen 2012; Lunn et al. 2015; Peterson et al. 2017; Uslu 2018). In der Lehrerprofessionsforschung wird damit an das „Konzept des Lehrerwissens“ angeschlossen. Um dieses zu rekonstruieren, werden Orientierungen oder subjektive Theorien von Lehrkräften erhoben und diese u.a. in epistemologische Überzeugungen operationalisiert (vgl. Neuweg 2014; Baumert und Kunter 2006, S. 496).

Das Forschungsfeld ,epistemologische Überzeugungen“ ist stark ausdifferenziert. Epistemologische Überzeugungen werden in einem Spannungsfeld konzeptualisiert, in dem Fragen nach der Dimensionierung, Domänen-, Themen- und Kontextabhängigkeit unterschiedlich beantwortet werden. Die Konturen der unterschiedlichen Konzeptualisierungsvorschläge werden im Folgenden kurz skizziert.

(1) Erwerb und Entwicklung epistemologischer Überzeugungen Epistemologische Überzeugungen werden unterschiedlich dimensionalisiert. Einerseits wird von der Eindimensionalität und andererseits von der Multidimensionalität epistemolo- 
gischer Überzeugungen ausgegangen. In Forschungsarbeiten zur Eindimensionalität epistemologischer Überzeugungen wird davon ausgegangen, dass die unterschiedlichen Facetten epistemologischer Überzeugungen sich nacheinander entwickeln. Demnach durchlaufen Subjekte unterschiedliche Entwicklungsstufen epistemologischer Überzeugungen: Es wird angenommen, dass Lern- sowie Reifungsprozesse, Bildungs- sowie Sozialisationserfahrungen und die Konfrontation mit evidenzbasierten Erkenntnissen, die beispielsweise den eigenen Wissensbeständen zuwiderlaufen, die Fortentwicklung der epistemologischen Überzeugungen beeinflussen (vgl. Kuhn et al. 2000; Kuhn und Weinstock 2009; Perry 1970). Jüngere Forschungsarbeiten negieren die linearen, eindimensionalen Entwicklungsmodelle; stattdessen werden epistemologische Überzeugungen multidimensional konzeptualisiert. Es wird die Ansicht vertreten, dass sich Dimensionen epistemologischer Überzeugungen unabhängig voneinander entwickeln können (vgl. Hofer 2001; Hofer und Pintrich 1997; Schommer 1990, 1998; Schommer-Aikins 2004, 2009; Schommer-Aikins et al. 2010). Beispielsweise legten Hofer und Pintrich ein mehrdimensionales Modell epistemologischer Überzeugungen vor, die sowohl in Abhängigkeit als auch in Unabhängigkeit von verschiedenen Wissensdomänen ausgebildet werden können. Sie unterscheiden die Bereiche „Nature of Knowledge“ (Sicherheit und Struktur des Wissens) und „Nature of Knowing“ (Quelle und Rechtfertigung des Wissens) (vgl. Hofer 2001; Hofer und Pintrich 1997).

\section{(2) Domänenabhängigkeit und -unabhängigkeit epistemologischer Überzeugun-} gen In Konzeptualisierungsvorschlägen epistemologischer Überzeugungen werden die Domänen unterschiedlich gewichtet. Im Rahmen der Ausbildung epistemologischer Überzeugungen sprechen einige Autoren den Domänen eine Bedeutung zu, während andere Autoren den Einfluss von Domänen bei der Entwicklung von Epistemologien als weniger bedeutsam beschreiben. Beide Positionen werden im Folgenden kurz konturiert. (a) Epistemologische Überzeugungen werden unabhängig von Wissensdomänen konzeptualisiert: Beispielsweise legen Schommer und Walker mit ihrer Untersuchung den Schluss nahe, dass sich ähnliche epistemologische Überzeugungen über unterschiedliche Wissensdomänen hinweg konstant halten. Schommer und Walker resümieren: „Epistemological beliefs in both domains predicted passage comprehension similarly, and most students showed a consistent level of epistemological sophistication across domains" (Schommer und Walker 1995, S. 424). (b) Epistemologische Überzeugungen werden in Abhängigkeit zu Wissensdomänen konzeptualisiert: Hierbei wird davon ausgegangen, dass in unterschiedlichen Wissensdomänen spezifische epistemologische Überzeugungen ausgebildet werden (vgl. Buehl et al. 2002; Op't Eynde et al. 2002).

(3) Weitere Einflussfaktoren auf epistemologische Überzeugungen Neuere Forschungsarbeiten brachten die Erkenntnis, dass auch die Thematik und deren Kontext Einfluss auf die Ausbildung von epistemologischen Überzeugungen nehmen. Das zog schließlich eine Modifikation der Modellierung epistemologischer Überzeugungen nach sich. Danach wird die Auffassung vertreten, wonach epistemologische Überzeugungen nicht nur domänen- und disziplinspezifisch, sondern auch kontextsowie themenspezifisch ausdifferenziert werden. In diesem Zusammenhang heben 
Mayer und Rosman hervor, dass die Vorstellung, epistemologische Überzeugungen ,als Traits im Sinne generalisierter, relativ zeit- und situationsstabiler Dispositionen“ (Mayer und Rosman 2016, S. 11) zu konzeptualisieren, obsolet sei. So wird von einem integrativen Ansatz ausgegangen, wonach Wissenstragende epistemologische Überzeugungen ausgebildet haben können, die domänenspezifisch sowie -unspezifisch verhaftet sind und je nach Kontext und Thematik variieren können (vgl. Hammer und Elby 2009; Lee et al. 2016; Muis et al. 2006; Schraw 2009; Stahl 2011; Trautwein et al. 2004). Der Einfluss des thematischen Kontextes bei der Ausprägung, Aktivierung und Tragfähigkeit epistemologischer Überzeugungen wird herausgestellt. In diesem Kontext wird auch die strenge, normativ aufgeladene Differenzierung zwischen naiven und sophistizierten, epistemologischen Überzeugungen kritisiert: So könne es in bestimmten Kontexten funktional sein, sich beispielsweise ausschließlich auf eine Theorie zu stützen und damit naive epistemologische Überzeugungen zu zeigen (vgl. Elby und Hammer 2010; Klopp und Stark 2016; Rosman et al. 2017). Auch Bromme et al. weisen im Kontext der ,kognitiven Arbeitsteilung" darauf hin, dass es in bestimmten Kontexten sinnhaft sein kann, sich auf das von Experten produzierte Wissen als Laien zu verlassen und damit streng genommen über naive epistemologische Überzeugungen zu verfügen (vgl. Bromme et al. 2008, 2016). Es besteht noch erheblicher Forschungsbedarf hinsichtlich der Frage, wie epistemologische Überzeugungen sich intra- und interdisziplinär sowie intraund interpersonell überlagern oder gar beeinflussen und wie diese Aspekte im Forschungsprozess aufgegriffen werden können (vgl. Limón 2006; Müller et al. 2008; Plöger-Werner 2015; Mayer und Rosman 2016).

Die bisherige Forschung zu epistemologischen Überzeugungen für den schulischen Kontext konzentrierte sich vor allem auf die Erforschung epistemologischer Überzeugungen in den MINT-Fächern (vgl. u.a. Depaepe et al. 2016; Dunekacke et al. 2016; Felbrich et al. 2008), während die geisteswissenschaftlichen Fächer bisher sowohl in der internationalen als auch in der nationalen Forschung weitgehend ausgespart wurden. Es liegen noch keine empirisch fundierten, theoretischen Konzeptualisierungen von epistemologischen Überzeugungen von Lehrkräften der geisteswissenschaftlichen Fächer vor. Bisherige Untersuchungen zu Überzeugungssystemen von Studierenden der Sprach- und Kulturwissenschaften zeigen lediglich, dass im Gegensatz zu jenen von Studierenden der Mathematik und der Ingenieurssowie Naturwissenschaften Wissen als deutlich relativer wahrgenommen wird (vgl. Trautwein et al. 2004). VanSledright und Maggioni (vgl. 2016) zeigen bzgl. des Fachs Geschichte, dass insbesondere die Objektivität und Genese historischen Wissens herausgestellt wird. Darüber hinaus lag der Forschungsfokus bisher vornehmlich auf den epistemologischen Überzeugungen, die Akteuren reflexiv zugänglich sind und entsprechend expliziert werden können. Untersuchungen legen allerdings nahe, dass gerade auch die impliziten handlungsleitenden Orientierungen eine für vermittelnde Unterrichtstätigkeiten eine Rolle spielen (vgl. Klopp und Stark 2017).

Auf diese Forschungsdesiderata reagiert die vorliegende Untersuchung, indem (a) die impliziten handlungsleitenden Überzeugungen von Lehrkräften in den Blick genommen werden, (b) Lehrkräfte der geisteswissenschaftlichen Fächer befragt werden und (c) die kollektiv geteilten Überzeugungen fokussiert werden. Da nur auf wenige Forschungsbefunde rekurriert werden kann, ist diese Untersuchung hypothesen- 
sowie theoriegenerierend angelegt. Die Forschungsfrage der vorliegenden Studie lautet vor dem Hintergrund der genannten Forschungsdesiderate damit weiter präzisiert: Welche handlungsleitenden, kollektiv geteilten, epistemologischen Überzeugungen haben Lehrkräfte der geisteswissenschaftlichen Fächerdomäne inkorporiert?

\section{Methodisches Vorgehen und methodologische Fundierung}

Das leitende Erkenntnisinteresse wurde mit Hilfe eines qualitativ-rekonstruktiven Zugangs bearbeitet (vgl. Bohnsack 2014, 2017), um neben den expliziten auch die impliziten, inkorporierten epistemologischen Überzeugungen herausarbeiten zu können. ${ }^{1}$ Im Folgenden wird das methodische Design skizziert.

Datenerhebung Als Datenerhebungsinstrument dienten Gruppendiskussionen (vgl. Loos und Schäffer 2001). Hiermit wird die „Möglichkeit der Rekonstruktion kollektiver Orientierungen, also des milieu- und kulturspezifischen Orientierungswissens" (Bohnsack et al. 2010, S. 7, Hervorhebung im Original) eröffnet: Die Gruppendiskussionsteilnehmenden können ihre kollektiv geteilten, handlungsleitenden Orientierungen aktualisieren und über ihre konjunktiven Erfahrungen eigenstrukturiert, selbstläufig sowie ohne propositionale Themensetzung seitens der Forschenden erzählen. Im Rahmen dieser Untersuchung wurden Lehrkräfte eines geisteswissenschaftlichen Faches (z.B. Deutsch, Geschichte, evangelische/ katholische Religionslehre) aufgefordert, von ihren Unterrichtserfahrungen zu erzählen. Über diese so generierten Erzählungen wurden die handlungsleitenden Überzeugungen bzw. Orientierungen der Lehrkräfte zu den epistemologischen Grundlagen ihres geisteswissenschaftlichen Faches rekonstruierbar. ${ }^{2}$

Datengrundlage Insgesamt wurden 19 Gruppendiskussionen geführt, die anhand eines ,theoretical samplings“ (vgl. Glaser und Strauss 2010) zusammengestellt wurden. Insgesamt haben an diesen Gruppendiskussionen 78 Lehrkräfte teilgenommen. Alle Lehrkräfte unterrichten mindestens ein geisteswissenschaftliches Fach an ei-

\footnotetext{
${ }^{1}$ Im Kontext der methodologischen Fundierung der Erhebungsmethode Dokumentarische Methode werden Wissensbestände, unter denen auch sensu Neuweg (vgl. 2014) epistemologische Überzeugungen gefasst werden, auch als Orientierungen (Orientierungsschemata bzw. -rahmen) spezifiziert. Da in dieser Untersuchung epistemologische Überzeugungen als eine Facette von (Lehrer-)Wissen ausgewiesen werden, wird im fortlaufenden Text der Terminus „Orientierungen“, „Orientierungsschemata“ bzw. „Orientierungsrahmen“ als Synonyme für epistemologische Überzeugungen verwendet. Die Dokumentarische Methode unterscheidet aus einer erkenntnislogischen Sicht nicht zwischen subjektivem und objektivem Wissen. Mit ihr können sowohl habitualisierte Praktiken in Form von handlungspraktischem Wissen als auch explizierbares, kommunikatives Wissen unterschieden werden (vgl. Bohnsack 2014, 2017).

2 Kollektiv geteilte Orientierung zeigen sich insbesondere in interaktiv dichten Textpassagen, die auch als Fokussierungsmetaphern und „dramaturgische Höhepunkte“ (Bohnsack und Przyborski 2010, S. 234) ausgewiesen werden. Die befragten Akteure konzentrieren sich vornehmlich auf ein Erlebniszentrum und bearbeiten dieses Thema diskursiv in einer erzählenden, beschreibenden und bildhaft-sprachlichen Weise (vgl. Bohnsack 2014, 2017).
} 
ner weiterführenden Schule in der Sekundarstufe I und/oder II. ${ }^{3}$ Um die Sättigung des Samples auf der Basis unterschiedlicher Vergleichshorizonte zu gewährleisten, wurden folgende Samplingstrategien angewandt: Novizen- oder Experten-Status der Lehrkräfte (z. B. Referendarinnen und Referendare, Lehrkräfte mit langjähriger Berufserfahrung); unterschiedlicher Bildungshintergrund der Professionellen (z. B. abgeschlossene Promotion in einer geisteswissenschaftlichen Disziplin); unterrichtliche Tätigkeit im gleichen geisteswissenschaftlichen Fach (z. B. Gruppendiskussionen ausschließlich mit Deutschlehrkräften oder evangelischer Religionslehre); unterrichtliche Tätigkeit in unterschiedlichen geisteswissenschaftlichen Fächern (z. B. Gruppendiskussionen, in denen eine Vielfalt geisteswissenschaftlicher Fächer vertreten waren) und eine Datenerhebung in unterschiedlichen Bundesländern (um eine ggf. erkennbare Bedingtheit durch schulische Kontexte wie Lehrerbildungssystem oder Curricula sichtbar zu machen).

Datenauswertung Die in dieser Studie erhobenen Daten wurden mit der Dokumentarischen Methode (vgl. Bohnsack 2014, 2017) ausgewertet. Die mit dieser Methode verbundenen einzelnen Analyseschritte des empirischen Materials - die formulierende und die reflektierende Interpretation - operationalisieren das erkenntnislogische Potenzial dieses Zugangs. Methodologisch basieren die Analyseschritte auf einer wissenssoziologischen Leitdifferenz (vgl. Mannheim, 1964, 1980): Mit Hilfe der formulierenden Interpretation wird der immanente Sinngehalt herausgearbeitet und dargestellt, was Lehrkräfte über die Genese von geisteswissenschaftlichem Wissen und die damit verbundene Kulturvermittlung kommunizieren. Beispielsweise: Welche kulturbezogenen Inhalte nennen Lehrkräfte eines geisteswissenschaftlichen Faches als Unterrichtsgegenstände? Mit Hilfe der reflektierenden Interpretation wird über die Frage, wie Lehrkräfte kommunizieren, der exmanente Sinn herausgearbeitet. Durch diesen reflektierenden Analyseschritt werden die atheoretischen, konjunktiven, inkorporierten, impliziten, handlungsleitenden sowie erfahrungsbasierten Wissensbestände, Überzeugungen bzw. Orientierungsrahmen rekonstruiert. Beispielsweise: Wie erzählen Lehrkräfte über die Vermittlung von Wissensbeständen im geisteswissenschaftlichen Unterricht?

Datenverdichtung und -generalisierung Mit der vorliegenden Untersuchung wurde ein Beitrag zur Theorieentwicklung im Kontext der Lehrerprofessionalität geisteswissenschaftlicher Fächer geleistet. Der Erhebungs-, Auswertungs- sowie Verdichtungsprozess der Daten wurde solange iterativ abgewechselt, bis keine neue Erkenntnisse mehr generiert werden konnten und eine Sättigung des theoretischen Samples belegt werden konnte. Die Ergebnisse wurden im Rahmen von komparativen Analysen sowie einer induktiven und insbesondere abduktiven Erkenntnishaltung (vgl. Meyer 2009) verdichtet und zur einer sinngenetischen Typenbildung generalisiert (vgl. Bohnsack 2013; Bohnsack und Nohl 2010; Nentwig-Gesemann 2013). Entsprechend der Standards qualitativer Sozialforschung (vgl. Bohnsack

\footnotetext{
3 Bzgl. der epistemologischen Gemeinsamkeiten geisteswissenschaftlicher Fächer vgl. Abschn. 1 dieses Beitrags.
} 
2005) wurden die Ergebnisse in zwei Forschungswerkstätten intersubjektiv sowie kommunikativ-konsensuell validiert. ${ }^{4}$

\section{Ergebnisse: Orientierungen von Lehrkräften zur Epistemologie der Geisteswissenschaften}

Welche handlungsleitenden Orientierungen hinsichtlich der Epistemologie der Geisteswissenschaften von Lehrkräften, die ein geisteswissenschaftliches Fach unterrichten, lassen sich rekonstruieren? Im Folgenden werden die empirischen Ergebnisse der Untersuchung dargestellt.

\subsection{Tertium Comparationis: Geltung}

In den Daten zeigte es sich, dass über alle Gruppendiskussionen hinweg die Lehrkräfte in ihren Erzählungen über ihren Unterricht darum ringen, welche Bedingungen geisteswissenschaftliche Wissensbestände erfüllen müssen, sodass sie über Geltung verfügen und entsprechend an Schülerinnen und Schüler weitergegeben werden können. Bei der Datenverdichtung und -generalisierung emergierte also „Geltung“ als gemeinsames Thema, das allen Fällen des Samples gemeinsam ist und sich als Tertium Comparationis der sinngenetischen Typenbildung darstellte.

Die Auseinandersetzung mit Geltung konnte anhand von fünf Vergleichshorizonten spezifiziert werden:

1. Genese von Sinn

2. Sicherheit der generierten Erkenntnis und Grenzen der Erkenntnis

3. Objektbezug: Konstituierung des geisteswissenschaftlichen Erkenntnisgegenstandes

4. Subjekt-Subjekt-Bezug: Lehrkräfte sowie Schülerinnen und Schüler im Kontext von wissensgenerierenden Verfahren

5. Zielstellungen im Rahmen geisteswissenschaftlichen Arbeitens

Die Orientierungen wurden $\mathrm{zu}$ einer sinngenetischen Typenbildung verdichtet. Diese besteht wiederum aus drei Idealtypen, die unterschiedliche Modi im Umgang mit und in der Herstellung von Geltung aufzeigen. Im Folgenden werden die einzelnen Idealtypen und die sie konstituierenden Herstellungspraxen konturiert, wobei der Fokus auf dem Vergleichshorizont „Genese von Sinn“ von Kulturobjekten liegt (vgl. zu einer weitergehenden Darstellung hinsichtlich der Vergleichshorizonte zwei bis fünf Rau 2020a, b).

\footnotetext{
4 Ich danke den beiden Forschungswerkstätten unter der Leitung von Frau Prof. Dr. Annette Scheunpflug und Herrn Dr. Nikolaus Schröck, in deren Rahmen ich wertvolle Impulse für die Fort- und Weiterentwicklung dieser Untersuchung erhalten habe und meine Untersuchungsergebnisse validieren konnte.
} 


\subsection{Der Modus eines dogmaorientierten Kontingenzstopps (Typ 1)}

Dieser Idealtyp ist apriorisch dahingehend fixiert, dass den im Unterricht thematisierten kulturellen Objektivationen eine einzige Bedeutung zugesprochen wird, dem Schülerinnen und Schüler im Rahmen dessen Dechiffrierung nachspüren sollen. Diese Orientierung zieht einen Erkenntnismonismus nach sich. Diese Orientierung an einer apriorischen Fixierung auf eine einzige, eindeutige Bedeutung von Kulturobjekten geht mit einem bestimmten erkenntnislogischen Verfahren einher: Lehrkräfte dechiffrieren kulturelle Objektivationen, indem sie diese in ihren historischen Entstehungskontext einbetten und vor eben diesem Hintergrund decodieren. Dabei wird die vermeintliche Intentio auctoris des jeweiligen Erkenntnisobjektes herausgearbeitet. Eine weitere Orientierung dieses Idealtyps zeigt ferner, dass dogmatisch verfahren und Kontingenz abgewehrt wird: Anhand eines etablierten erkenntnislogischen Routinemusters werden immer wieder die gleichen Wissensbestände über Kulturobjekte reproduziert. Nachfolgender Ausschnitt einer Gruppendiskussion zeigt die entsprechende handlungsleitende Orientierung, wonach Kulturobjekte monoperspektivisch interpretiert werden und an ihre Historizität angebunden werden. Die Gruppe erzählt von Franz Kafkas „Die Verwandlung“. Die Erzählung sei im ortsansässigen Theater inszeniert worden. Die Lehrkräfte heben hervor, dass sie die Inszenierung des Regisseurs ablehnen, da dieser die literarische Vorlage nicht entlang der (vermeintlichen) Autorenintention umgesetzt habe. Die Passage rundet den Diskurs der Gruppe Wangerooge ab: Zuvor haben sich die Lehrkräfte über die (Nicht-)Notwendigkeit ausgetauscht, wissenschaftliche Erkenntnisse rezipieren und diese an Schülerinnen und Schüler im Unterricht vermitteln zu müssen. Des Weiteren tauschen sie sich über die Schwierigkeit von vor allem jüngeren Schülerinnen und Schüler aus, sich geisteswissenschaftliche Inhalte zu erschließen. Ein weiteres Thema sind die Interpretationsleistungen von Schülerinnen und Schüler, die die Notengebung erschweren: Denn die Lehrkräfte täten sich schwer damit, Schülerinterpretationen als zulässige Interpretation auszuweisen. Des Weiteren unterhalten sich die Lehrkräfte über Unterrichtsinhalte, die Schülerinnen und Schüler im Kontext von wissenschaftspropädeutischen Seminaren erwerben sollten.

\section{Gruppe Wangerooge, Passage ,Normative Lesart‘, Zeile 822-859}

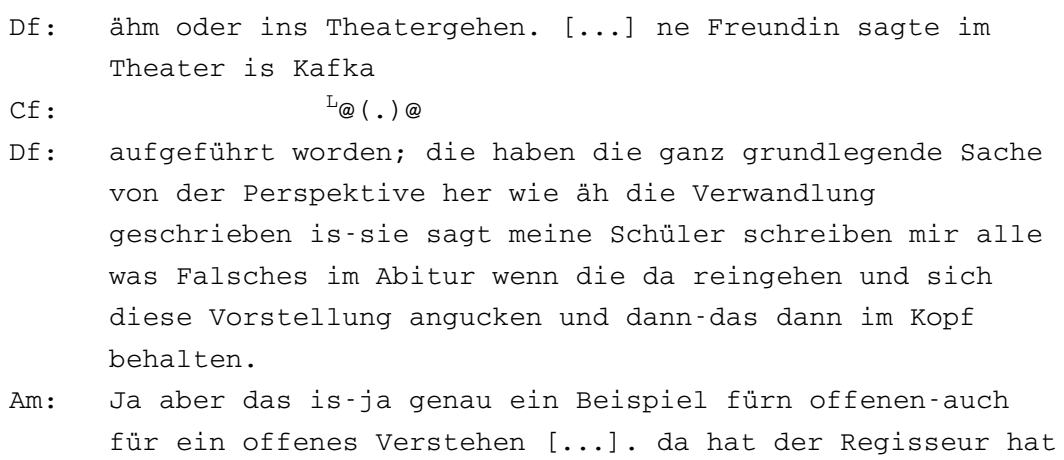




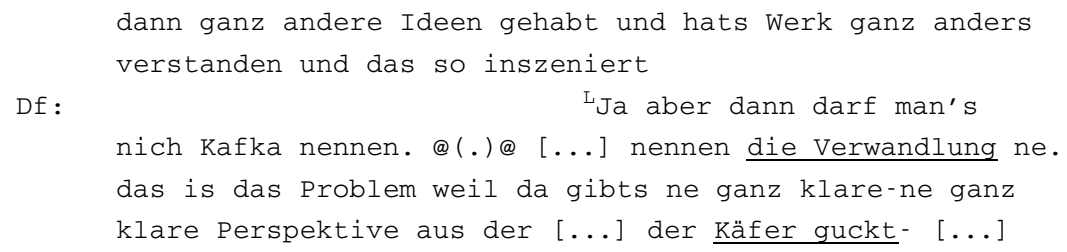

Die Äußerungen von Df werden im weiteren Diskurs weiterausgeführt und kommunikativ sowie konsensuell validiert. Die für den Typ 1 vorgestellten Orientierungen spiegeln sich u. a. auch in den Gruppen Norderney sowie Rügen wieder. Im vorliegenden empirischen Material zeigt sich folgendes: Zwar erkennen die Lehrkräfte an, dass literarische Vorlage unterschiedlich inszeniert werden können (,,Ja aber das is-ja genau ein Beispiel fürn offenen-auch für ein offenes Verstehen“). Dessen ungeachtet zeigt sich aber für ihr Professionsverständnis, dass die Bedeutungsvielfalt von Kulturobjekten in der unterrichtlichen Handlungspraxis nicht aufgegriffen wird. So wird letztlich die vom Regisseur gebotene Inszenierungsleistung sowie die damit verbundene Interpretation des literarischen Textes seitens der Gruppe negiert: So entspräche die Inszenierung nicht einer historizitätsorientierten, monoperspektivischen Lesart bzw. der Autorenintention, die die Lehrkräfte der Gruppe Wangerooge präferieren. Das Kulturobjekt „Die Verwandlung“ gebe im Verständnis der Lehrkräfte „ne ganz klare Perspektive aus der [...] der Käfer guckt“ vor. Kulturrezipierende sowie -inszenierende können zwar andere Lesarten haben: Gleichwohl entsprechen diese Lesarten dann aber nicht mehr dem vom Autor der kulturellen Objektivation eingeschriebenen Lesart. So holt Am beispielsweise ins Wort, dass der Regisseur das „Werk ganz anders verstanden“ und ,ganz andere Ideen gehabt“ hat. Entgegnet wird, dass diese Inszenierung man dann eben „nich Kafka nennen“ dürfe.

Der geisteswissenschaftliche Charakter wird Interpretations- und Inszenierungsleistungen, die nicht einem an Historizität orientierten, monoperspektivischen erkenntnislogischen Zugang folgen, abgesprochen. In diesen Orientierungen dokumentiert sich, dass die Lehrkräfte die eine Bedeutung kultureller Objektivationen gespeichert halten und für ihren Unterricht reaktivieren. Sie orientieren sich am historischen Entstehungskontext des Kulturguts, dessen Bedeutungsgehalt nicht erweitert wird. ${ }^{5}$

\subsection{Der Modus einer applikationsorientierten Konstruktivismusermöglichung (Typ 2)}

Der Typ 2 zeichnet sich dadurch aus, dass kulturellen Objektivationen ein Bedeutungs- und Sinngewebe zugeschrieben wird. Während Typ 1 am Erkenntnismonismus orientiert ist und Erkenntnisobjekte auf Basis ihres Produktionskontexts ver-

\footnotetext{
5 Diese Handlungspraxis schließt an die hermeneutische Theoriegeschichte des 19. Jahrhunderts an: So hebt zum Beispiel Ast hervor, dass ,das [...] Verstehen [...] ein wahrhaftes Reproduzieren oder Nachbilden des schon Gebildeten“ (Ast 1976, S. 120) sei (vgl. hierzu auch Schleiermacher 1977 [1838]; Dilthey 1973 [1907-1910]). Vor diesem Hintergrund werden nicht alle Facetten abgebildet, die innerhalb des Diskurses moderner hermeneutischer Theoriebildung geführt wurden (vgl. hierzu ausführlich Rau 2020a).
} 
standen werden, dokumentiert sich in Typ 2 eine Orientierung am Erkenntnispluralismus. Dabei wird die Bedeutung von kulturellen Objektivationen auf der Basis des Rezeptionskontextes nachgezeichnet. Mehrdeutig aufgefasste Themen werden in ihrem Sinngewebe decodiert. Dadurch werden das Sinngewebe bzw. die Bedeutungszuschreibungen von kulturellen Objektivationen stetig erweitert. Alle schulischen Akteurinnen und Akteure sind gleichberechtigt in die Bedeutungsverleihung inkludiert. Darüber hinaus wird deutlich, dass dieser Typ an der Applikation als Form der Vermittlung orientiert ist: Schülerinnen und Schüler werden explizit aufgefordert, kulturellen Objektivationen vor dem Hintergrund ihrer eigenen Erfahrungswelt zu begegnen und diese entsprechend zu dechiffrieren. Dem geisteswissenschaftlichen Unterricht wird ein Mehrwert für die erkennenden Subjekte attestiert: Diese sollen auf Basis applikationsorientierter erkenntnislogischer Dechiffrierungsverfahren Impulse für die Gestaltung der eigenen Erfahrungs- und Lebenswelt gewinnen. Verstehensleistungen, Bedeutungszuschreibungen und Dechiffrierungen führen so $\mathrm{zu}$ einem prinzipiell unabschließbaren Erkenntnisprozess. Zugleich ist in diese Orientierungen von Lehrkräften auch ein dualistisch-erkenntnislogischer Zugang inkorporiert: Lehrkräfte relationieren sich hier in einem asymmetrischen Verhältnis zu ihren Schülerinnen und Schülern, indem sie anhand epistemologischer Grundprinzipien (z. B. Begründung von Lesarten, Wissenschaftsorientierung usw.) das Bedeutungsangebot von Kulturobjekten einschätzen und Lesarten als ,gültig“ bzw. ,nichtgültig" ausweisen. Anhand einer Passage der Gruppe Amrum, in der sich über Johann Wolfgang Goethes „Faust“ unterhalten wurde, wird Typ 2 sichtbar. Weitere Themen der Gruppe Amrum, die der nachfolgenden Passage vorausgehen, sind: Abgrenzung des geisteswissenschaftlichen Fächerkanons gegenüber dem naturwissenschaftlichen, Kompetenzen zur Erschließung von kulturellen Objektivationen, Lernziele des geisteswissenschaftlichen Unterrichts und unterrichtliche Herausforderungen als Lehrkräfte des geisteswissenschaftlichen Fächerkanons. Die von Cm beschriebene Orientierung wird im Diskurs konsensuell und kommunikativ validiert und in weiteren Passagen auch in anderen Gruppen - wie z. B. Hiddensee und Usedom - ebenfalls zum Ausdruck gebracht.

Gruppe Amrum, Passage, Mehrwert von Lektüre‘, Zeile 1844-1855




Zum einen ist für Typ 2 orientierend, dass die biographische und soziokulturelle Erfahrungswelt von Schülerinnen und Schülern die erkenntnislogische Basis darstellt und kulturellen Objektivationen vor diesem Hintergrund Bedeutung zugeschrieben werden. Im vorausgegangenen empirischen Beispiel tauschen sich die Lehrkräfte über „Faust“ und dessen Mehrwert für die Persönlichkeitsentwicklung von Schülerinnen und Schülern aus. Zunächst wird das mit dem literarischen Text verwobene Motiv herausgearbeitet. Anschließend wird die Verbindung zwischen den literarischen Motiven und dem Rezeptionskontext der Erkenntnissubjekte konturiert. So sei der „Faust“ „,auch n Angebot über dich selber zu nachzudenken“. In dieser Passage und weiteren (vgl. z. B. Zeile 482-810) dokumentiert sich ferner: Die Einbettung kultureller Objektivationen in den Rezeptionskontext von Schülerinnen und Schülern wird als unabdingbar für den Verstehensprozess verstanden. Die Lehrkräfte begegnen der Differenz zwischen Produktions- und Rezeptionskontext der kulturellen Objektivation, indem der literarische Text auf die Lebenssituation von Schülerinnen und Schülern sowie auf deren mögliche Entwicklungsoptionen bezogen wird. Cm betont, dass ,der Faust neben der ganzen Wissenschaftlichkeit auch $\mathrm{n}$ Angebot [sei,] über dich nachzudenken [...] ey was will ich eigentlich aus meinem Leben" machen. Mit einer solchen Fragehaltung wird der Gegenwartsbezug des Textes immer wieder aufs Neue hergestellt und Schülerinnen und Schüler werden aufgefordert, sich über den Text mit sich selbst zu befassen. Zusammenfassend kann festgehalten werden: Typ 1 ist durch die Orientierung an als tradierungswürdig angesehenen Bedeutungszuschreibungen gekennzeichnet. Für Typ 2 ist orientierend, dass Rezipierende persönlich und individuell von kulturellen Objektivationen angesprochen werden: Da sich Rezeptionskontexte immer wieder verändern, kann das Sinngewebe von Kulturobjekten niemals vollständig erschlossen werden; stattdessen muss das Sinngewebe immer weitergesponnen werden. Kulturellen Objektivationen wird immer wieder eine neue Bedeutung verliehen. Im Rahmen dieser erkenntnisgenerierenden Verfahren loten die Erkenntnissubjekte selbst aus, welche Lesarten Geltung beanspruchen.

\subsection{Der Modus einer fundierungsorientierten Pluralismusästimation (Typ 3)}

Für Typ 3 ist Folgendes kennzeichnend: Zum einen wird eine Orientierung an zu vermittelnden Wissensbeständen über die Geschichte der Geisteswissenschaften erkennbar, sodass potenziell die historische Entwicklung geisteswissenschaftlicher Inhalte seitens der Schülerinnen und Schüler nachvollzogen werden können sollte. Diese Wissensbestände sind epochal strukturiert geordnet. Gleichzeitig wird eine Orientierung an analytischen Wissensbeständen erkennbar: Schülerinnen und Schüler sollen für die gesellschaftliche Bedeutung von kulturellen Objektivationen sensibilisiert werden. Zum anderen ist für diesen Typ die Inkorporation eines zirkulären Erkenntnisprozesses und die Bedeutung eines Erkenntnispluralismus charakteristisch: Für dasselbe Erkenntnisobjekt sollen immer wieder neue Erkenntnisse bzw. Dechiffrierungsmöglichkeiten generiert werden. Orientierungen dieses Typs erweitern das Sinngewebe von kulturellen Objektivationen mittels vielfältiger Decodierungen. Es werden multiperspektivische und multivalente Erkenntniszugänge herangezogen, um den Kulturobjekten immer wieder neue Lesarten zu entnehmen. In 
diesem Zusammenhang wird der Subjektivität von Schülerinnen sowie Schülern und dem Verweisungskontext bzw. -horizont eine hohe Bedeutung im Erkenntnisprozess beigemessen. Die Geltung von Dechiffrierungsleistungen ist nach diesen Orientierungen dann gegeben, wenn Schülerinnen und Schüler ihre Verstehensleistungen im Kontext von Kommunikationsgemeinschaften expliziert, diskutiert und intersubjektiv validiert haben. Zur empirischen Illustration des Typs 3 dient die folgende Passage: Die Lehrkräfte tauschen sich über eine Unterrichtseinheit aus, in der das Thema „Befreiungstheologie“ besprochen wurde. Dm erzählt, dass er gemeinsam mit seiner Frau eine Unterrichtseinheit entworfen hat, welche sowohl an einem musischen als auch an einem naturwissenschaftlichen Gymnasium durchgeführt wurde. Weitere Themen der Gruppen Föhr sind: zu vermittelnde Wissensbestände zum Nachvollzug historischer Abläufe, der Mehrwert geisteswissenschaftlicher Inhalte für Schülerinnen und Schüler, das (vermeintlich) geringe Interesse von Schülerinnen und Schüler an geisteswissenschaftlichen Themen. Die von Dm aufgeworfene Orientierung wird im weiteren Diskurs von den anderen Lehrkräften elaboriert und letztlich kommunikativ sowie konsensuell validiert. Auch in den Gruppen Amrum, Fehmarn, Hiddensee sowie Ummanz zeigt sich die nachfolgende, empirisch illustrierte Orientierung:

\section{Gruppe Föhr, Passage ,Multiperspektive Erschließung‘, Zeile 1147-1219}

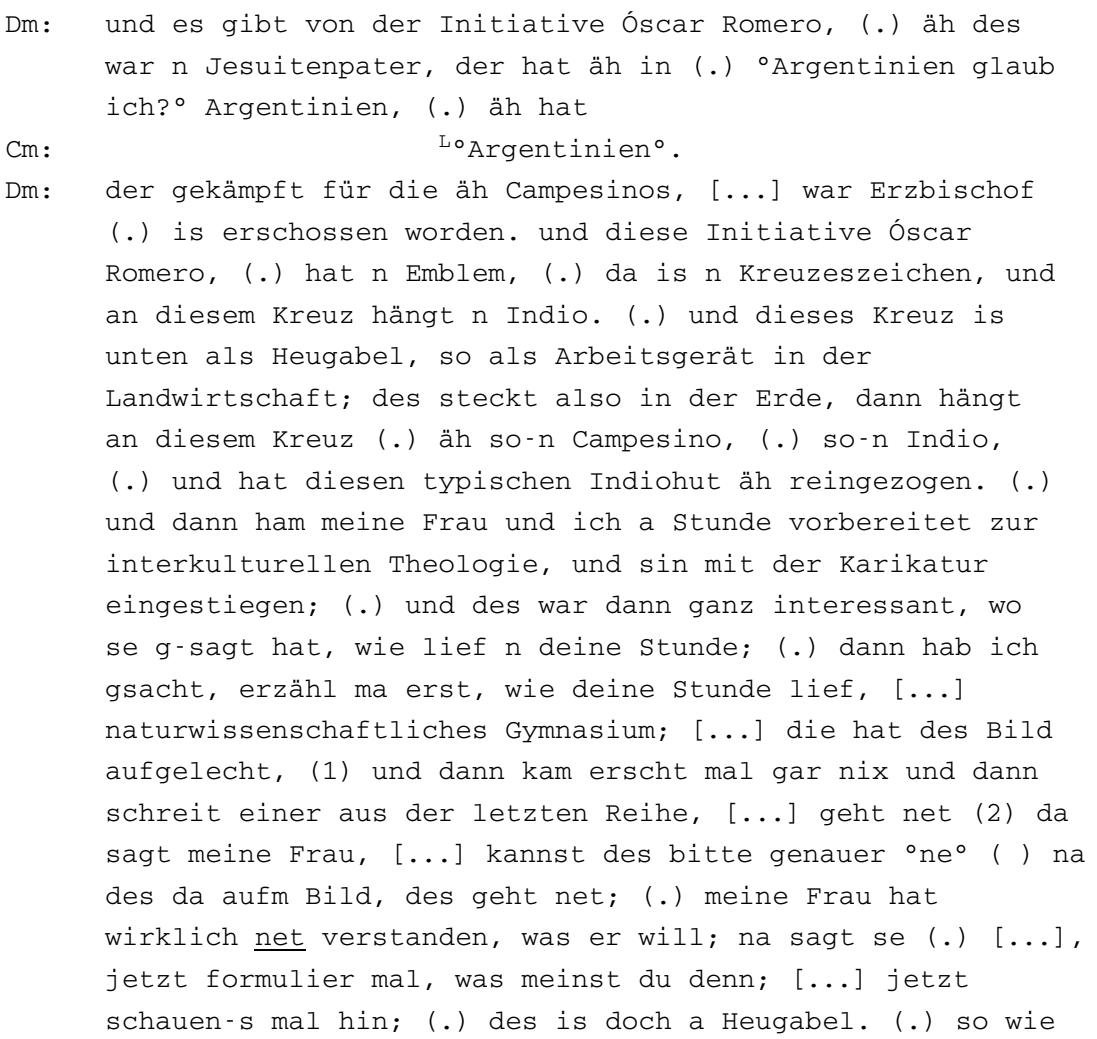




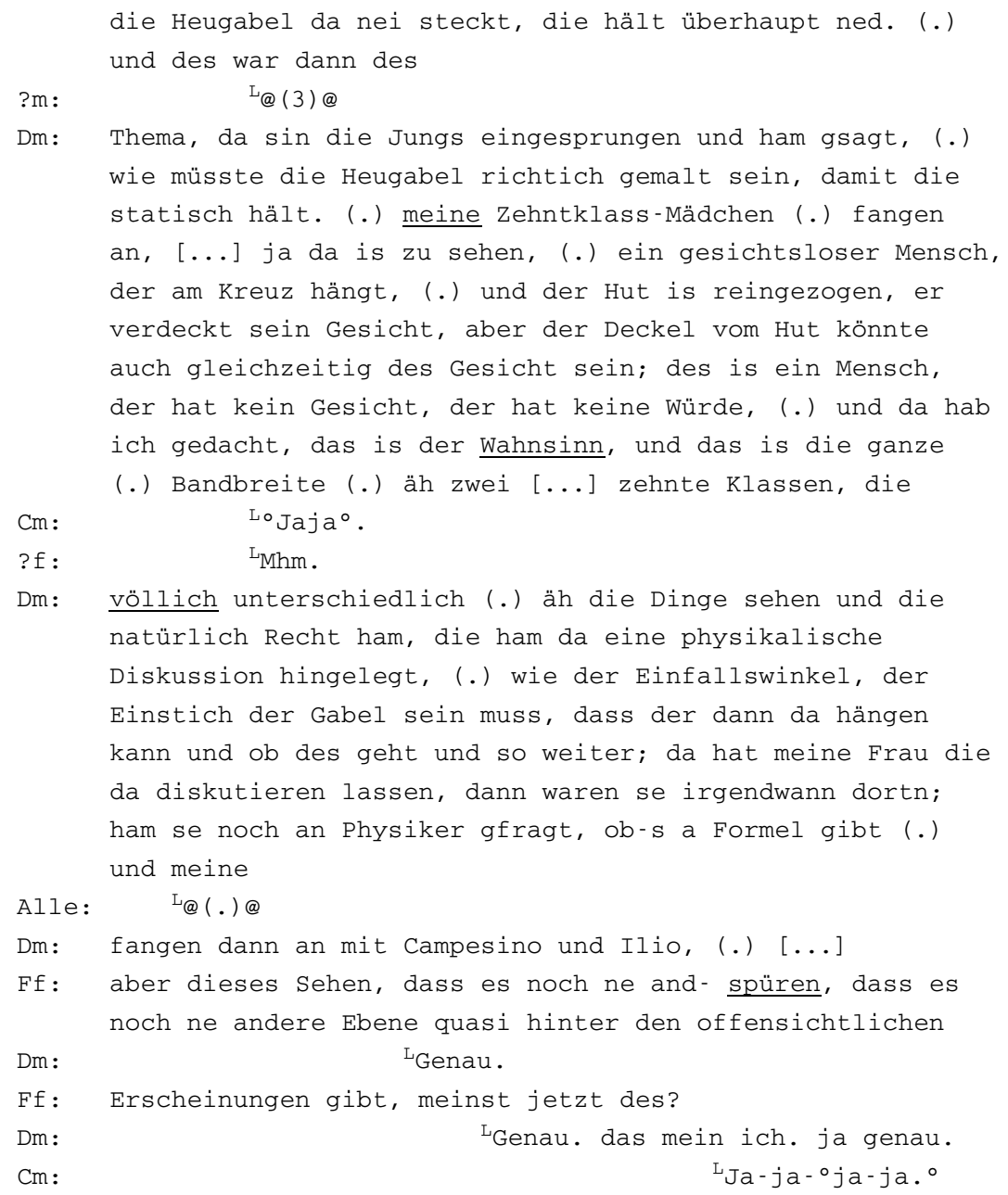

In dieser Passage, in der sich die Lehrkräfte über eine Unterrichtseinheit im Kontext interkultureller Theologie bzw. der Befreiungstheologie am Beispiel der Initiative Óscar Romero unterhalten, dokumentiert sich die Aufgeschlossenheit der Lehrkräfte gegenüber unterschiedlichen Decodierungen von kulturellen Objektivationen im geisteswissenschaftlichen Unterricht. Die Decodierung wird an die Subjektivität der Schülerinnen und Schüler gebunden. Zwar sei der Unterrichtseinstieg für die beiden Klassen mit derselben „Karikatur“ begonnen worden, allerdings zeigten die beiden Klassen unterschiedliche Reaktionen: Die Schülerinnen und Schüler der naturwissenschaftlichen Klasse monierten, dass der Einstichwinkel der auf der Karikatur abgebildeten Heugabel vom Produzierenden dieses Kulturobjekts nicht korrekt gesetzt worden sei. Im Gegensatz hierzu wählten die Schülerinnen und Schüler des musischen Gymnasiums einen anderen Zugang zu ebendiesem Erkenntnisobjekt: Sie verstehen die auf der Karikatur abgebildete Person als ein Abbild für ein Kollektivsubjekt, dass sinnbildlich für die Entrechteten und Ausgebeuteten bzw. die 
entrechteten und ausgebeuteten Campesinos steht. Beide Klassen zeigen beim selben Kulturobjekt unterschiedliche Verstehensleistungen. In den Orientierungen der Lehrkräfte dokumentiert sich, dass beide Lesarten Gültigkeit besitzen. Gleichzeitig dokumentiert sich, dass die Subjektivität bzw. die subjektiven Wissensbestände der Schülerinnen und Schüler als unabdingbar für die Erkenntnisgenese gelten. Bei der Decodierung von Kulturobjekten wird der Subjektivität der Schülerinnen und Schüler bzw. deren subjektiven Wissensbeständen ein indispensabler Anteil beigemessen; sie sind unabdingbar für die Erschließung der Erkenntnisobjekte. Das kann mit den Äußerungen von Dm belegt werden, die von den anderen Gruppendiskussionsteilnehmenden validiert werden: ,das ist der Wahnsinn, und das is die ganze (.) Brandbreite (.) äh zwei zehntk- zehnte Klassen, die [...] völlich unterschiedlich (.) äh die Dinge sehen und die natürlich Recht ham“. Die Decodierungsleistungen bieten darüber hinaus wichtige Impulse für die weitere Unterrichtsgestaltung und den fachlichen Austausch: So lässt die Lehrkraft die Schülerinnen und Schüler, die den dargestellten Einstichwinkel der Heugabel unter physikalischen Gesichtspunkten beanstandet haben, eine ,physikalische Diskussion“ führen. Die Passage endet mit der Aufforderung der Lehrkräfte an ihr eigenes unterrichtliches kulturvermittelndes Handeln, dass Schülerinnen und Schüler für ein ,,Sehen, [...] [ein] spüren“ sensibilisiert werden müssten, „,dass es noch ne andere Ebene quasi hinter den offensichtlichen [...] Erscheinungen gibt“". Pointiert zeichnen sich die Orientierungen von Lehrkräften des Typs 3 dahingehend aus, dass unterschiedliche Decodierungsfolien herangezogen werden, um das Sinngewebe äquivoker Kulturobjekte zu erschließen. Der Subjektivität und den subjektiven Wissensbeständen der Schülerinnen und Schülern wird ein indispensabler Anteil bei der Erkenntnisgenese zugesprochen. Gleichzeitig wird in diesen Orientierungen die Verantwortung erkennbar, fachwissenschaftliche Wissensbestände (z. B. kulturtheoretische Inhalte, historische Entwicklung geisteswissenschaftlicher Inhalte) an Schülerinnen und Schüler zu vermitteln, sodass eine Begegnung mit kulturellen Objektivationen möglich ist.

\section{Diskussion}

Im Folgenden werden die Befunde dieser Studie theoretisch kontextualisiert. Hierbei wird aufgezeigt, dass die Orientierungen zur Epistemologie der Geisteswissenschaften kulturrezipierendend orientiert sind (vgl. Abschn. 6.1). Zudem werden die Orientierungen hinsichtlich ihrer Nähe zu einem begrenzten Verständnis kultureller Objektivationen sowie zu weltgesellschaftlichen Ansprüchen (vgl. Abschn. 6.2) diskutiert. Es werden Überlegungen zur weiteren Forschung angestellt (vgl. Abschn. 6.3).

\subsection{Orientierung an Kulturrezeption}

In den rekonstruierten Orientierungen von Lehrkräften zur Epistemologie der Geisteswissenschaften sowie in der idealtypischen Verdichtung derselben wurde ersichtlich, dass Lehrkräfte in ihren Orientierungen um die Erlangung von Geltung geisteswissenschaftlicher Inhalte ringen. Dabei wurde anhand der Vergleichsdimension „Genese von Sinn“ gezeigt, wie in den einzelnen Idealtypen die Bedeutung von 
kulturellen Objektivationen decodiert wird. Der Begründungs- sowie Rechtfertigungszusammenhang von Bedeutungszuschreibungen ist in den einzelnen Idealtypen unterschiedlich ausdifferenziert: Für Typ 1 ist eine historisierend monoperspektivische sowie deduktive Decodierungslogik charakteristisch. Typ 2 zeichnet sich durch Orientierungen aus, in denen kulturelle Objektivationen an den biographischen Erfahrungskontext von Schülerinnen und Schülern angebunden werden. Für Typ 3 ist bezeichnend, dass das Sinngewebe von Kulturobjekten für die Lehrkräfte nicht festgelegt ist, sondern in vielerlei Perspektive als möglich wahrgenommen wird. Damit wird aber auch deutlich: Kultur wird ausschließlich im Modus der Rezeption begegnet. Empirisch konnten keine Orientierungen an der Schaffung von kulturellen Objektivationen festgestellt werden.

\subsection{Orientierungsmuster, Überlieferungsformen und weltgesellschaftliche Ansprüche}

Die Orientierungen an geisteswissenschaftlicher Epistemologie manifestieren „eine Überlieferungs- und Vergegenwärtigungsform des kulturelles Sinns“ (Assmann 1992, S. 21). Konkretisieren sich diese Orientierungen im Unterricht, dann tragen sie dazu bei, dass Schülerinnen und Schüler ,,in ihrer jeweiligen Kultur heimisch [werden], sie bleiben nicht Fremde im eigenen symbolischen Umfeld" (Fend 2008, S. 49). Alle Typen orientieren sich an epochal geordneten Wissensbeständen und könnten damit potenziell einen Beitrag dazu leisten, Schülerinnen und Schüler mit der Geschichte der abendländisch-geisteswissenschaftlichen Entwicklungen vertraut zu machen. Der geisteswissenschaftliche Fachunterricht wird damit zu einem „Ort des Wissens [...] [, in dem] die Auslegung der fundierenden Texte“ (Assmann 1992, S. 87) in den Blick genommen wird. Dadurch sind die ,Repetition und Interpretation [...] funktional äquivalente Verfahren in der Herstellung kultureller Kohärenz"“ (Assmann 1992, S. 89, Hervorhebung im Original). Durch die fortwährende Explikation eines Sinns bzw. eines Sinngewebes von kulturellen Objektivationen und die Inanspruchnahme von Geltung dieser Sinnzuschreibungen wird die Bedeutung kultureller Objektivationen sowohl gespeichert als auch reaktiviert und fortgeführt.

In Abschn. 6.1 wurde ausgeführt, dass sich die Lehrkräfte im Kontext epistemologischer Grundfragen im geisteswissenschaftlichen Unterricht an der Kulturrezeption orientieren und bereits existierende Kulturobjekte in ihrem Bedeutungsgehalt dechiffriert werden. Die Befunde zeigen, dass Lehrkräfte das Sinnpotenzial von Kulturobjekten in unterschiedlichem Ausmaß ausschöpfen: Die Spannbreite reicht von eindeutig bis $\mathrm{zu}$ mehrdeutig gefassten kulturellen Objektivationen und damit verbundenen sinngenerierenden Verfahren.

In den empirischen Ergebnissen zeigt sich, dass in geisteswissenschaftlichen Fächern ein ,differenzierungstheoretischer Kulturbegriff“ handlungsleitend wird: Die kulturellen Objektivationen beziehen sich ,,auf jene intellektuellen und künstlerischen Ausdeutung[en], die einer normativen Ausdeutung würdig erscheinen“" (Reckwitz 2012, S. 79), und damit gleichzeitig als (Hoch-)Kultur ein ausdifferenziertes Teilsystem von Gesellschaft widerspiegeln (vgl. Reckwitz 2004). Exemplarisch wird das in den illustrierten Textpassen deutlich, wenn Kafkas „Verwandlung“, Goethes „Faust“ oder das Emblem der Campesinos epistemologisch erschlossen werden. Die 
Orientierungen der Lehrkräfte lassen sich mit Reckwitz als ,ein institutionalisierter ,Treuhänder' der Symbolisierungen einer Gesellschaft, die hier reproduziert, aber auch neu produziert werden“ (Reckwitz 2012, S. 81) interpretieren. Dieser Befund überrascht insofern, als dass sich hier in den Orientierungen von Lehrkräften die kulturellen Hybridisierungsprozesse von Gesellschaften (vgl. Bhabha 2000; Welsch 2012) wenig spiegeln und die Forderung nach einer Orientierung an einem bedeutungsorientierten Kulturbegriff (vgl. Reckwitz 2004) und nach der Vielfalt kultureller Objektivationen auf wenig Resonanz stößt sowie kaum Orientierungen an der Dekonstruktion „eigener Kultur“ bzw. der Beobachtung von Kultur (vgl. Scheunpflug et al. 2012) zu finden sind. Die transkulturelle Leerstelle, die fehlende Orientierung an kulturbezogener Kontingenz und damit fehlende Anschlussperspektiven an weltgesellschaftliche Entwicklungen werden erkennbar. Daraus dürften sich unmittelbare Konsequenzen für die Lehrerbildung ergeben.

\subsection{Weitere Forschungsperspektiven}

Die Reflexion der epistemologischen Grundlagen des Faches, das Lehrkräfte unterrichten, stellt eine Facette von Lehrerprofessionalität dar. Für die geisteswissenschaftlichen Fächer ist hier noch ein weitgehendes Desiderat festzustellen. Mit der vorgestellten qualitativen Studie wurden nun Typen epistemologischer Orientierungen vorgelegt. Der nächste Schritt müsste es nun sein, diese Typen in Kategorien und Items so zu übersetzen, dass eine quantitative Untersuchung sichtbar macht, in welchen Häufigkeiten sich diese Orientierungen finden lassen. Eine Aufgabe zukünftiger Forschung wird es also sein, die Forschungsergebnisse dieses hypothesengenerierenden-rekonstruktiven Beitrags mit den Konzeptualisierungen epistemologischer Überzeugungen, die weitgehend auf quantitativen Untersuchungsdesigns fußen, zusammenzuführen.

Funding Open Access funding enabled and organized by Projekt DEAL.

Open Access Dieser Artikel wird unter der Creative Commons Namensnennung 4.0 International Lizenz veröffentlicht, welche die Nutzung, Vervielfältigung, Bearbeitung, Verbreitung und Wiedergabe in jeglichem Medium und Format erlaubt, sofern Sie den/die ursprünglichen Autor(en) und die Quelle ordnungsgemäß nennen, einen Link zur Creative Commons Lizenz beifügen und angeben, ob Änderungen vorgenommen wurden.

Die in diesem Artikel enthaltenen Bilder und sonstiges Drittmaterial unterliegen ebenfalls der genannten Creative Commons Lizenz, sofern sich aus der Abbildungslegende nichts anderes ergibt. Sofern das betreffende Material nicht unter der genannten Creative Commons Lizenz steht und die betreffende Handlung nicht nach gesetzlichen Vorschriften erlaubt ist, ist für die oben aufgeführten Weiterverwendungen des Materials die Einwilligung des jeweiligen Rechteinhabers einzuholen.

Weitere Details zur Lizenz entnehmen Sie bitte der Lizenzinformation auf http://creativecommons.org/ licenses/by/4.0/deed.de.

\section{Literatur}

Assmann, J. (1992). Das kulturelle Gedächtnis: Schrift, Erinnerung und politische Identität in frühen Hochkulturen. München: C. H. Beck. https://doi.org/10.17104/9783406703409. 
Ast, F. (1976). Hermeneutik. In H.-G. Gadamer \& G. Boehm (Hrsg.), Seminar: Philosophische Hermeneutik (S. 111-130). Frankfurt a. M.: Suhrkamp.

Baumert, J., \& Kunter, M. (2006). Stichwort: Professionelle Kompetenz von Lehrkräften. Zeitschrift für Erziehungswissenschaft, 9(4), 469-520. https://doi.org/10.1007/s11618-006-0165-2.

Bhabha, H. K. (2000). Die Verortung der Kultur (Mit einem Vorwort von Elisabeth Bronfen. Deutsche Übersetzung von Michael Schiffmann und Jürgen Freudl). Tübingen: Stauffenburg.

Böhme, G. (1994). Einführung in die Philosophie: Weltweisheit - Lebensform - Wissenschaft. Frankfurt a. M.: Suhrkamp.

Bohnsack, R. (2005). Standards nicht-standardisierter Forschung in den Erziehungs- und Sozialwissenschaften. Zeitschrift für Erziehungswissenschaft, 7(4), 63-81. https://doi.org/10.1007/978-3-32280769-4_5.

Bohnsack, R. (2013). Typenbildung, Generalisierung und komparative Analyse: Grundprinzipien der dokumentarischen Methode. In R. Bohnsack, I. Nentwig-Gesemann \& A.-M. Nohl (Hrsg.), Die dokumentarische Methode und ihre Forschungspraxis: Grundlagen qualitativer Sozialforschung (3. Aufl., S. 241-270). Wiesbaden: Springer. https://doi.org/10.1007/978-3-531-19895-8_11.

Bohnsack, R. (2014). Rekonstruktive Sozialforschung: Einführung in qualitative Methoden (9. Aufl.). Opladen: Barbara Budrich.

Bohnsack, R. (2017). Praxeologische Wissenssoziologie. Opladen: Barbara Budrich.

Bohnsack, R., \& Nohl, A.-M. (2010). Komparative Analyse und Typenbildung in der dokumentarischen Methode. In G. Cappai, S. Shimada \& J. Straub (Hrsg.), Interpretative Sozialforschung und Kulturanalyse: Hermeneutik und die komparative Analyse kulturellen Handelns (S. 101-128). Bielefeld: transcript. https://doi.org/10.14361/9783839407936-003.

Bohnsack, R., \& Przyborski, A. (2010). Diskursorganisation, Gesprächsanalyse und die Methode der Gruppendiskussion. In R. Bohnsack, A. Przyborski \& B. Schäffer (Hrsg.), Das Gruppendiskussionsverfahren in der Forschungspraxis (2. Aufl., S. 233-248). Opladen: Barbara Budrich. https://doi.org/10. 2307/j.ctvddzp5w.17.

Bohnsack, R., Przyborski, A., \& Schäffer, B. (2010). Einleitung: Gruppendiskussionen als Methode rekonstruktiver Sozialforschung. In R. Bohnsack, A. Przyborski \& B. Schäffer (Hrsg.), Das Gruppendiskussionsverfahren in der Forschungspraxis (2. Aufl., S. 7-22). Opladen: Barbara Budrich. https:// doi.org/10.2307/j.ctvddzp5w.3.

Brenner, P. J. (2011a). Sinn. In H. Reinalter \& P. J. Brenner (Hrsg.), Lexikon der Geisteswissenschaften: Sachbegriffe - Disziplinen - Personen (S. 738-744). Wien: Böhlau. https://doi.org/10.7767/boehlau. 9783205790099.738 .

Brenner, P. J. (2011b). Hermeneutik/Interpretation/Verstehen. In H. Reinalter \& P. J. Brenner (Hrsg.), Lexikon der Geisteswissenschaften. Sachbegriffe - Disziplinen - Personen (S. 314-323). Wien: Böhlau. https://doi.org/10.7767/boehlau.9783205790099.314.

Bromme, R., Kienhues, D., \& Stadtler, M. (2016). Die kognitive Arbeitsteilung als Herausforderung für die Forschung zu epistemischen Überzeugungen. In A.-K. Mayer \& T. Rosman (Hrsg.), Denken über Wissen und Wissenschaft: Epistemologische Überzeugungen (S. 25-38). Lengerich: Pabst Science Publishers.

Bromme, R., Kienhues, D., \& Stahl, E. (2008). Knowledge and epistemological beliefs: an intimate but complicate relationship. In M.S. Khine (Hrsg.), Knowing, knowledge and beliefs: epistemological studies across diverse cultures (S. 423-441). New York: Springer. https://doi.org/10.1007/978-14020-6596-5_20.

Bråten, I. (2016). Epistemic cognition interventions: issues, challenges, and directions. In J. A. Greene, W. A. Sandoval \& I. Bråten (Hrsg.), Handbook of epistemic cognition (S. 360-371). New York: Routledge.

Buehl, M.M., \& Fives, H. (2016). The role of epistemic cognition in teacher learning and praxis. In J. A. Greene, W. A. Sandoval \& I. Bråten (Hrsg.), Handbook of epistemic cognition (S. 247-264). New York: Routledge.

Buehl, M. M., Alexander, P.A., \& Murphy, P. K. (2002). Beliefs about schooled knowledge: domain specific or domain general? Contemporary Educational Psychology, 27(3), 415-449. https://doi.org/10. 1006/ceps.2001.1103.

Cornelißen, C. (2011). Objektivität. In H. Reinalter \& P. J. Brenner (Hrsg.), Lexikon der Geisteswissenschaften: Sachbegriffe - Disziplinen - Personen (S. 595-602). Wien: Böhlau. https://doi.org/10.7767/ boehlau.9783205790099.595.

Depaepe, F., de Corte, E., \& Verschaffel, L. (2016). Mathematical epistemological beliefs. In J. A. Greene, W. A. Sandoval \& I. Bråten (Hrsg.), Handbook of epistemic cognition (S. 147-164). New York: Routledge. 
Dilthey, W. (1973). Der Aufbau der geschichtlichen Welt in den Geisteswissenschaften. Stuttgart: Teubner.

Dunekacke, S., Jenßen, L., Eilerts, K., \& Blömeke, S. (2016). Epistemological beliefs of prospective preschool teachers and their relation to knowledge, perception, and planning abilities in the field of mathematics: a process model. ZDM: Mathematics Education, 48, 125-137. https://doi.org/10.1007/ s11858-015-0711-6.

Elby, A., \& Hammer, D. (2010). Epistemological resources and framing: a cognitive framework for helping teachers interpret and respond to their students' epistemologies. In L. D. Bendixen \& F.C. Feucht (Hrsg.), Personal epistemology in the classroom: theory, research, and implications for practice (S. 409-434). Cambridge: Cambridge University Press. https://doi.org/10.1017/ CBO9780511691904.013.

Felbrich, A., Müller, C., \& Blömeke, S. (2008). Epistemological beliefs concerning the nature of mathematics among teacher educators and teacher education students in mathematics. ZDM: Mathematics Education, 40(5), 763-776. https://doi.org/10.1007/s11858-008-0153-5.

Fend, H. (2008). Neue Theorie der Schule: Einführung in das Verstehen von Bildungssystemen (2. Aufl.). Wiesbaden: VS. https://doi.org/10.1007/978-3-531-91788-7_2.

Glaser, B. G., \& Strauss, A. L. (2010). Grounded Theory: Strategien qualitativer Forschung (3. Aufl., aus dem Amerikanischen von Axel T. Paul und Stefan Kaufmann. Mit einem Geleitwort von Bruno Hildebrand). Bern: Huber.

Hammer, D., \& Elby, A. (2009). On the form of a personal epistemology. In B. K. Hofer \& P. R. Pintrich (Hrsg.), Personal epistemology: the psychology of beliefs about knowledge and knowing (S. 169-190). New York: Routledge.

Hofer, B.K. (2001). Personal epistemology research: implications for learning and teaching. Journal of Educational Psychology Review, 13(4), 353-383. https://doi.org/10.1023/A:1011965830686.

Hofer, B.K., \& Bendixen, L.D. (2012). Personal epistemology: theory, research, and future directions. In K. R. Harris, S. Graham \& T.C. Urdan (Hrsg.), APA educational psychology handbook: theories, constructs, and critical issues (S. 227-256). Washington, D.C.: American Psychological Association. https://doi.org/10.4324/9780203424964.

Hofer, B.K., \& Pintrich, P. R. (1997). The development of epistemological theories: beliefs about knowledge and knowing and their relation to learning. Review of Educational Research, 67(1), 88-140. https://doi.org/10.3102/00346543067001088.

Klopp, E., \& Stark, R. (2016). Persönliche Epistemologien - Elemente wissenschaftlicher Kompetenz. In A.-K. Mayer \& T. Rosman (Hrsg.), Denken über Wissen und Wissenschaft: Epistemologische Überzeugungen (S. 39-69). Lengerich: Pabst Science Publishers.

Klopp, E., \& Stark, R. (2017). Quantitative und qualitative Analysen zur Validität und Reliabilität des Fragebogens zur Erfassung epistemischer Überzeugungen (FEE). In A. Bernholt, H. Gruber \& B. Moschner (Hrsg.), Wissen und Lernen. Wie epistemische Überzeugungen Schule, Universität und Arbeitswelt beeinflussen (S. 39-58). Münster: Waxmann.

Kuhn, D., \& Weinstock, M. (2009). What is epistemological thinking and why does it matter? In B. K. Hofer \& P.R. Pintrich (Hrsg.), Personal epistemology: the psychology of beliefs about knowledge and knowing (S. 121-144). New York: Routledge.

Kuhn, D., Cheney, R., \& Weinstock, M. (2000). The development of epistemological understanding. Cognitive Development, 15(3), 309-328. https://doi.org/10.1016/S0885-2014(00)00030-7.

Lee, C. D., Goldmann, S. R., Levine, S., \& Magliano, J. (2016). Epistemic cognition in literary reasoning. In J. A. Greene, W. A. Sandoval \& I. Bråten (Hrsg.), Handbook of epistemic cognition (S. 165-183). New York: Routledge.

Limón, M. (2006). The domain generality-specificity of epistemological beliefs: a theoretical problem, a methodological problem or both? International Journal of Educational Research, 45(1-2), 7-27. https://doi.org/10.1016/j.ijer.2006.08.002.

Loos, P., \& Schäffer, B. (2001). Das Gruppendiskussionsverfahren: Theoretische Grundlagen und empirische Anwendung. Opladen: Leske + Budrich. https://doi.org/10.1007/978-3-322-93352-2.

Lunn, J., Walker, S., \& Mascardi, J. (2015). Personal epistemological and teaching. In H. Fives \& M. G. Gill (Hrsg.), International handbook of research on teachers' beliefs (S. 319-335). New York: Routledge.

Mannheim, K. (1964). Wissenssoziologie: Auswahl aus dem Werk. Berlin: Luchterhand.

Mannheim, K. (1980). Strukturen des Denkens. Frankfurt a. M.: Suhrkamp.

Mayer, A.-K., \& Rosman, T. (2016). Epistemologische Überzeugungen und Wissenserwerb in akademischen Kontexten. In A.-K. Mayer \& T. Rosman (Hrsg.), Denken über Wissen und Wissenschaft: Epistemologische Überzeugungen (S. 7-23). Lengerich: Pabst Science Publishers. 
Meyer, M. (2009). Abduktion, Induktion - Konfusion: Bemerkungen zur Logik der interpretativen Sozialforschung. Zeitschrift für Erziehungswissenschaft, 12(3), 302-320. https://doi.org/10.1007/s11618009-0067-1.

Muis, K. R., Bendixen, L. D., \& Haerle, F. C. (2006). Domain-generality and domain-specificity in personal epistemology research: philosophical and empirical reflections in the development of a theoretical framework. Educational Psychology Review, 18, 3-54. https://doi.org/10.1007/s10648-006-9003-6.

Müller, S., Paechter, M., \& Rebmann, K. (2008). Aktuelle Befunde zur Lehr-Lernforschung: Epistemologische Überzeugungen zu Wissen und Wissenserwerb. bwp@. Berufliche Lehr-/Lernprozesse - Zur Vermessung der Berufsbildungslandschaft, 14, 1-17.

Nentwig-Gesemann, I. (2013). Die Typenbildung der dokumentarischen Methode. In R. Bohnsack, I. Nentwig-Gesemann \& A.-M. Nohl (Hrsg.), Die dokumentarische Methode und ihre Forschungspraxis: Grundlagen qualitativer Sozialforschung (3. Aufl., S. 295-323). Wiesbaden: Springer. https://doi. org/10.1007/978-3-531-19895-8_13.

Neuweg, G. H. (2014). Das Wissen der Wissensvermittler. Problemstellungen, Befunde und Perspektiven der Forschung zum Lehrerwissen. In E. Terhart, H. Bennewitz \& M. Rothland (Hrsg.), Handbuch der Forschung zum Lehrerberuf (S. 583-614). Münster: Waxmann.

Op't Eynde, P., de Corte, E., \& Verschaffel, L. (2002). Framing students' mathematics-related beliefs: a quest for conceptual clarity and a comprehensive categorization. In G.C. Leder, E. Pehkonen \& G. Torner (Hrsg.), Beliefs: a hidden variable in mathematics education? (Mathematics Education Library Bd. 31, S. 13-37). New York: Kluwer Academic Publishers. https://doi.org/10.1007/0-30647958-3_2.

Perry, W.G. (1970). Forms of intellectual and ethical development in the college years: a scheme. New York: Holt, Rinehart and Winston.

Peterson, E. G., Alexander, P. A., \& Lis, A. (2017). The argument for epistemic competence. In A. Bernholt, H. Gruber \& B. Moschner (Hrsg.), Wissen und Lernen: Wie epistemische Überzeugungen Schule, Universität und Arbeitswelt beeinflussen (S. 255-270). Münster: Waxmann.

Plöger-Werner, M. (2015). Epistemologische Überzeugungen von Erzieherinnen und Erziehern. Die Bedeutung für das pädagogische Handeln in Kindertageseinrichtungen. Wiesbaden: Springer. https:// doi.org/10.1007/978-3-658-09749-3.

Rau, C. (2020a). Kulturtradierung in geisteswissenschaftlichen Fächern. Eine rekonstruktive Studie zu epistemologischen Überzeugungen von Lehrkräften. Bad Heilbrunn: Verlag Julius Klinkhardt. https:// doi.org/10.35468/5811.

Rau, C. (2020b). Welche Sicherheitsansprüche zeigen sich in den epistemologischen Überzeugungen von Lehrkräften eines geisteswissenschaftlichen Fachs? Eine empirische Rekonstruktion. In U. Hericks, M. Keller-Schneider, W. Meseth \& A. Rauschenberg (Hrsg.), Fachliche Bildung und Professionalisierung von Lehrerinnen und Lehrern (S. 95-110). Bad Heilbrunn: Julius Klinkhardt.

Reckwitz, A. (2004). Die Kontingenzperspektive der „Kultur“. Kulturbegriffe, Kulturtheorien und das kulturwissenschaftliche Forschungsprogramm. In F. Jaeger \& J. Rüsen (Hrsg.), Handbuch der Kulturwissenschaften (S. 1-20). Stuttgart: Metzler. https://doi.org/10.1007/978-3-476-00468-0_1.

Reckwitz, A. (2012). Die Transformation der Kulturtheorien. Zur Entwicklung eines Theorieprogramms: Mit einem Nachwort zur Studienausgabe 2006: Aktuelle Tendenzen der Kulturtheorien (3. Aufl.). Weilerswist: Velbrück Wissenschaft.

Reinalter, H., \& Brenner, P. J. (2011). Vorwort. In H. Reinalter \& P. J. Brenner (Hrsg.), Lexikon der Geisteswissenschaften: Sachbegriffe - Disziplinen - Personen (S. V-X). Wien: Böhlau. https://doi.org/10. 7767/boehlau.9783205790099.

Rosman, T., Mayer, A.-K., Kerwer, M., \& Krampen, G. (2017). The differential development of epistemic beliefs in psychology and computer science students: a four-wave longitudinal study. Learning and Instruction, 49, 166-177. https://doi.org/10.1016/j.learninstruc.2017.01.006.

Scheunpflug, A., Franz, J., \& Stadler-Altmann, U. (2012). Zur „Kultur“ in pädagogischen Zusammenhängen. In I. T. Fink, B. Hill, V.-I. Reinwand \& A. Wenzlik (Hrsg.), Die Kunst, über Kulturelle Bildung zu forschen: Theorie- und Forschungsansätze (Kulturelle Bildung Bd. 29, S. 99-109). München: kopaed.

Schleiermacher, F. (1977). Hermeneutik und Kritik. Mit einem Anhang sprachphilosophischer Texte Schleiermachers. Frankfurt a. M.: Suhrkamp. Herausgegeben und eingeleitet von Manfred Frank

Schommer, M. (1990). Effects of beliefs about the nature of knowledge on comprehension. Journal of Educational Psychology, 82(3), 498-504. https://doi.org/10.1037/0022-0663.82.3.498.

Schommer, M. (1998). The influence of age and education on epistemological beliefs. British Journal of Educational Psychology, 68, 551-562. https://doi.org/10.1111/j.2044-8279.1998.tb01311.x. 
Schommer, M., \& Walker, K. (1995). Are epistemological beliefs similar across domains? Journal of Educational Psychology, 87(3), 424-432. https://doi.org/10.1037/0022-0663.87.3.424.

Schommer-Aikins, M. (2004). Explaining the epistemological belief system: introducing the embedded systemic model and coordinated research approach. Educational Psychologist, 39(1), 19-29. https:// doi.org/10.1207/s15326985ep3901_3.

Schommer-Aikins, M. (2009). An evolving theoretical framework for an Epistemological belief system. In B. K. Hofer \& P. R. Pintrich (Hrsg.), Personal epistemology: the psychology of beliefs about knowledge and knowing (S. 103-118). New York: Routledge.

Schommer-Aikins, M., Bird, M., \& Bakken, L. (2010). Manifestations of an epistemological belief system in preschool to grade twelve classrooms. In L.D. Bendixen \& F.C. Feucht (Hrsg.), Personal epistemology in the classroom: theory, research, and implications for practice (S. 31-54). Cambridge: Cambridge University Press. https://doi.org/10.1017/CBO9780511691904.002.

Schraw, G. (2009). Knowledge: structures and processes. In P. A. Alexander \& P. H. Winne (Hrsg.), Handbook of educational psychology: second edition (S. 245-263). New York: Routledge.

Stahl, E. (2011). The generative nature of epistemological judgments: focusing on interactions instead of elements to understand the relationship between epistemological beliefs and cognitive flexibility. In J. Elen, E. Stahl, R. Bromme \& G. Clarebout (Hrsg.), Links between beliefs and cognitive flexibility: lessons learned (S. 37-60). Dordrecht: Springer. https://doi.org/10.1007/978-94-007-1793-0_3.

Trautwein, U., Lüdtke, O., \& Beyer, B. (2004). Rauchen ist tödlich, Computerspiele machen aggressiv? Allgemeine und theorienspezifische epistemologische Überzeugungen bei Studierenden unterschiedlicher Fachrichtungen. Zeitschrift für Pädagogische Psychologie, 18(3/4), 187-199. https://doi.org/ 10.1024/1010-0652.18.34.187.

Uslu, O. (2018). Analysis of variables that affect teaching learning approaches and epistemological beliefs of pre-service teachers by structural equation model. International Journal of Research in Education and Science, 4(1), 237-251. https://doi.org/10.21890/ijres.383167.

VanSledright, B., \& Maggioni, L. (2016). Epistemic cognition in history. In J. A. Greene, W. A. Sandoval \& I. Bråten (Hrsg.), Handbook of epistemic cognition (S. 128-146). New York: Routledge.

Veraart, A., \& Wimmer, R. (1995). Hermeneutik. In J. Mittelstraß (Hrsg.), Enzyklopädie Philosophie und Wissenschaftstheorie. Bd. 2: $\mathrm{H}-\mathrm{O}$ (S. 85-90). Stuttgart: J. B. Metzler.

Welsch, W. (2012). Was ist eigentlich Transkulturalität? In D. Kimmich \& S. Schahadat (Hrsg.), Kulturen in Bewegung: Beiträge zur Theorie und Praxis der Transkulturalität (S. 25-40). Bielefeld: transcript. https://doi.org/10.14361/transcript.9783839417294.25. 